template copy number from RNA data owing to B cell subtype specific variation in the expression of the $\mathrm{B}$ cell receptor. Hypothetically, however, RNA input based monitoring could be advantageous both owing to reduced input requirements and superior ability to detect $\mathrm{B}$ call malignancies of plasmablast and plasma cell origin, where the BCR is robustly expressed. Here we compared the ability of RNA and DNA based IGH chain sequencing to detect Burkitt's Lymphoma cell lines and Chronic Lymphocytic Leukemia samples at a frequency of $10^{-6}$ from peripheral blood.

Materials and Methods Here we present performance for rare clone detection utilizing the Ion Oncomine ${ }^{\mathrm{TM}}$ BCR IGH-SR assay and the Ion Oncomine ${ }^{\mathrm{TM}}$ BCR IGH-LR assay. These assays use multiplex primers targeting all known IGH germline variable genes in the framework 1 (FR1) or framework 3 (FR3) regions of the $\mathrm{B}$ cell receptor using either DNA or RNA as input. To evaluate detection sensitivity of the IGH-SR assay we utilized DNA or RNA from Burkitt's lymphoma cell lines as well as clinical chronic lymphocytic leukemia (CLL) samples controllably added to a background of peripheral blood leukocytes (PBL) by mass ratio to create specimens with a known target B cell frequency. Automated downsampling analysis was used to confirm libraries were sequenced to saturation. Library preparation and analysis was performed in replicate to quantify sensitivity of detection.

Results For each cell line, we prepared and sequenced (1) 30 libraries derived from amplification of $2 \mathrm{ug}$ gDNA spiked with 2pg cell line gDNA and (2) 10 libraries derived from amplification of $100 \mathrm{ng}$ RNA spiked with $0.1 \mathrm{pg}$ cell line total RNA. The Burkitt's lymphoma cell line and CLL samples were detected in 10/30 and 8/30 libraries respectively, consistent with the performance of orthologous DNA-based sequencing approaches. For RNA libraries, the Burkitt's lymphoma and CLL samples were detected in each library (10/10 and 10/10, respectively).

Conclusions Here we demonstrate the ability to detect B cell clones down to $10^{-6}$ from gDNA and RNA inputs utilizing the Ion Oncomine ${ }^{\mathrm{TM}}$ BCR IGH-SR assay. Feasibility for rare clone detection is shown in gDNA or RNA enabling $\mathrm{B}$ cell minimal residual disease research, and high sensitivity characterization the $\mathrm{B}$ cell role in response to checkpoint blockade within the tumor microenvironment. Importantly, we find that RNA based IGH sequencing may significantly reduce input requirements for rare clone detection, potentially enabling routine detection of clones at $10^{-6}$ frequency from a single library.

Disclosure Information G.M. Lowman: A. Employment (full or part-time); Significant; ThermoFisher Scientific. L. Pickle: A. Employment (full or part-time); Significant; ThermoFisher Scientific. M. Toro: A. Employment (full or part-time); Significant; ThermoFisher Scientific. J. Chang: A. Employment (full or part-time); Significant; ThermoFisher Scientific. D. TopacioHall: A. Employment (full or part-time); Significant; ThermoFisher Scientific. T. Looney: A. Employment (full or parttime); Significant; ThermoFisher Scientific.

\section{P05.02 AN INTEGRATED VIRTUAL TISSUE PLATFORM FOR INCORPORATING EXERCISE ONCOLOGY INTO IMMUNOTHERAPY}

A Hagar*, J Aponte Serrano. Indiana University Bloomington, Bloomington, IN, USA
We introduce a novel in silico platform for simulating solid tumor growth and anti-tumor immune response. We present the model, test the sensitivity and robustness of its parameters, and calibrate it with pre-clinical and clinical data from exercise oncology experiments which offer a natural biological backdrop for modulation of anti-tumor immune response. We then perform two virtual experiments with the model that demonstrate its usefulness in guiding pre-clinical and clinical studies on immunotherapy. The first virtual experiment probes the intricate dynamics in the tumor microenvironment between the tumor and the infiltrating immune cells. Such dynamics is difficult to probe during a pre-clinical study as it requires significant of redundancy in lab animals and is time and labor intensive. The result is a time series of spatiotemporal observational 'windows' into the tumor microenvironment that can serve as a platform to test several mechanistic hypotheses on the role and dynamics of different immune cells in ant-tumor immune response. The second virtual experiment shows how dosage and frequency of immunotherapy drugs can be optimized based on the aerobic fitness of the patient, so that possible adverse side effects of the treatment can be minimized.

Disclosure Information A. Hagar: None. J. Aponte Serrano: None.

\section{P06 Cell Therapy in Solid Tumors}

\section{P06.01 BISPECIFIC ANTIBODY-DRIVEN SYNTHETIC AGONISTIC RECEPTOR - TRANSDUCED T CELLS MEDIATE SPECIFIC AND CONDITIONAL THERAPY IN MELANOMA CANCER MODELS}

${ }^{1} \mathrm{M}$ Benmebarek*, 'J Keyl, ${ }^{1} \mathrm{~F}$ Märkl, ${ }^{2} \mathrm{M}$ Geiger, ${ }^{1} \mathrm{C}$ Karches, 'S Rausch, ${ }^{1} \mathrm{~A}$ Gottschlich, ${ }^{1} \mathrm{~A}$ Öner, ${ }^{1} \mathrm{M}$ Feinendegen, ${ }^{1} \mathrm{~J}$ Dörr, ${ }^{1} \mathrm{~B}$ Cadilha, ${ }^{1} \mathrm{~S}$ Endres, ${ }^{2} \mathrm{C}$ Klein, ${ }^{1} \mathrm{~S}$ Kobold. 'Division of Clinical Pharmacology, Munich, Germany; ${ }^{2}$ Roche Innovation Centre Zurich, Schlieren, Switzerland

\subsection{6/jitc-2020-ITOC7.80}

Background Immunotherapeutic approaches, including immune checkpoint blockade and adoptive T cell therapy (ACT) in the form of tumor-infiltrating lymphocytes (TILs), have had marked success in the treatment of melanoma. Despite these successes, many patients are refractory to treatment or relapse with therapy-resistant disease. To overcome these limitations, we propose a controlled ACT approach, where T cells are armed with synthetic agonistic receptors (SARs) that are conditionally activated only in the presence of a target melanomaassociated antigen, and a cross-linking bispecific antibody (BiAb) specific for both (SAR) T cell and tumour cell.

Materials and Methods A SAR composed of an extracellular EGFRvIII, trans- membrane CD28, and intracellular CD28 and $\mathrm{CD} 3 \mathrm{z}$ domains was fused via overlap- extension PCR cloning. T cells were retrovirally transduced to stably express our SAR construct. We validated our approach in two murine as well as two human cancer models expressing our melanoma-associated target antigens TYRP (murine) and MCSP (human). We confirmed conditional and specific stimulation and proliferation of our $\mathrm{T}$ cells, as well as their tumour-antigen-directed cytotoxicity, in vitro and in vivo.

Results Crosslinking TYRP-EGFRvIII (murine) and MCSPEGFRvIII (human) BiAb, monovalently selective for our SAR, induced conditional antigen-dependent activation, proliferation 